\title{
Self-reported Prospective Memory After Stroke
}

Dr. Christy Hogan, PhD, School of Applied Psychology, Menzies Health Institute

Queensland, Mt Gravatt, Griffith University. https://orcid.org/0000-0001-7325-9695

Associate Professor Petrea Cornwell, PhD, School of Allied Health Sciences, Menzies Health Institute Queensland, Griffith University; The Prince Charles Hospital Metro North Hospital and Health Service. https://orcid.org/0000-0003-2621-8713

Professor Jennifer Fleming, PhD, School of Health and Rehabilitation Sciences, University of Queensland. https://orcid.org/0000-0002-5603-2410

Professor David W. K. Man, Department of Rehabilitation Sciences, The Hong Kong Polytechnic University. https://orcid.org/0000-0002-6248-4712

Professor David H. K. Shum, PhD, Department of Rehabilitation Sciences, The Hong Kong Polytechnic University; School of Applied Psychology, Gold Coast, Griffith University. https://orcid.org/0000-0002-4810-9262 


\begin{abstract}
Prospective Memory (PM) is the memory for completing future intentions. This study aimed to compare self-reported PM of individuals with stroke to healthy controls, to determine if PM is impaired after stroke. Additionally, self-reported PM for individuals with stroke was compared to significant-other reports, ascertaining a level of self-awareness of PM function. Twenty-eight individuals with stroke, 25 significant-others, and 27 healthy controls completed the Brief Assessment of PM (BAPM) and the Prospective and Retrospective Memory Questionnaire (PRMQ) as part of a larger study. Individuals with stroke reported significantly more Basic Activities of Daily Living (BADL) PM failures compared to controls on Part A of the BAPM. On Part B, individuals with stroke reported BADL PM failures to be less problematic/important than controls, suggesting a lack of self-awareness into the consequences of PM failure. Individuals with stroke also reported significantly more PM and RM failures compared to controls on the PRMQ. No significant differences were found between individuals with stroke and their significant-others on both the BAPM and PRMQ. Results of this study helped to clarify the previous research and highlighted that individuals with stroke reported more PM failures than controls but underestimated the importance of such memory lapses.
\end{abstract}

Key words: Brain injury, Stroke, Prospective Memory, Self-report 


\section{Self-reported Prospective Memory After Stroke}

\section{Introduction}

Changes in self-awareness are common after brain injury, such as stroke, sometimes leaving individuals unaware of their deficits (Caldwell et al., 2014; Leung \& Liu, 2011). Additionally, individuals and their families commonly report impairments in memory function after stroke, but previous research has focused on retrospective memory (RM; memory for past information or events) rather than prospective memory (PM; Andrews et al., 2014; de Haan, Nys, \& Van Zandvoort, 2006; Kalashnikova, Zueva, Pugacheva, \& Korsakova, 2005). PM is the encoding, storage, and delayed retrieval of intended actions (Shum, Levin, \& Chan, 2011).

Intact PM is necessary to complete many everyday activities (e.g., remembering to pay a bill before the due date or remembering to take medication at a certain time). PM impairment can have detrimental effects on an individual's independence (Groot, Wilson, Evans, \& Watson, 2002; Shum, Fleming, \& Neulinger, 2002) including basic (BADL) and instrumental activities of daily living (IADL; Fish, Wilson, \& Manly, 2010; Man, Yip, Lee, Fleming, \& Shum, 2015; Woods, Weinborn, Velnoweth, Rooney, \& Bucks, 2012).

Assessment of PM usually involves self-report or behavioural measurement. While self-reported methods have their limitations (Uttl \& Kibreab, 2011), there are also advantages such as efficiency in clinical administration and serving as a brief screening tool before comprehensive PM assessment is prescribed . Specific strengths of self-report methods would be a better understanding of an individuals' own perspective of how frequently PM lapses occur, as well as how important they think these lapses are, which could be useful for clientcentred rehabilitation (Ekstam, Uppgard, Kottorp, \& Tham, 2007; Hartman-Maeir, Soroker, Ring, \& Katz, 2002; Leung \& Liu, 2011; Ownsworth \& Clare, 2006). In addition, these 
questionnaires also provide a way to collect the opinions of others about a patient's PM ability, allowing comparison between the patient and significant-other scores, providing an indication of an individual's self-awareness of his or her PM ability. While self-reported PM measures do not directly assess PM performance in an observable way, they can still provide useful information for clinicians/researchers working with clinical populations.

Compared to other types of memory (i.e., RM), PM research is relatively new, only gaining interest in the 1970s (Kliegel, McDaniel, \& Einstein, 2008). There is a paucity of research on PM after stroke and mixed findings have been indicated when examining PM using self-report methods. A recent scoping review (Hogan, Fleming, Cornwell, \& Shum, 2016) reported that the majority of the self-report literature (three studies out of five) found that individuals with stroke did not report any more or less PM problems than healthy controls. For example, Brooks, Rose, Potter, Jayawardena, and Morling (2004) used a 10item questionnaire about real-life PM and reported no differences in self-reported PM between individuals with stroke $(n=25)$ and controls $(n=25)$. However, this measure did not have any reported psychometric properties. The authors concluded that individuals with stroke were unaware of their PM impairments as other PM measures showed impairments when compared to controls. It was further suggested that individuals with stroke may have been reporting their pre-stroke PM function, as the questionnaire that was used included some questions that referred to PM situations that the patients would not have encountered in the rehabilitation ward.

An intervention study (Miller \& Radford, 2014) used Part A of the Comprehensive Assessment of PM (CAPM; Shum \& Fleming, 2014) which consists of 39 items and assesses the frequency of PM lapses in ADLs. Individuals with stroke $(n=40)$ and significant-others $(n=32)$ reported similar PM forgetting and the scores fell within the normal limits indicating no PM impairment. However, when individuals with stroke were assessed at baseline using a 
clinical PM measure (Royal Prince Alfred Prospective Memory Test; Radford, Lah, Say, \& Miller, 2011), they were found to perform more than one $S D$ below the normal mean, indicating PM impairment.

Utilising the Prospective and Retrospective Memory Questionnaire (PRMQ; Smith, Del Sala, Logie, \& Maylor, 2000), which asks individuals to rate how often everyday minor memory mistakes occur to them for both prospective and retrospective memory items, Kim, Craik, Luo, and Ween (2009) found that both individuals with stroke $(n=12)$ and controls $(n$ $=12$ ) reported similar levels of lapses in both RM and PM. Both groups reported PM abilities close to the normative population average and because the stroke sample had relatively subtle memory problems, impairments may not have been detected by the questionnaire. However, consistent with previous research, when the sample was assessed using a clinical PM measure, the Virtual Week (Rendell \& Henry, 2009), impairments were found, particularly for time-based PM (i.e., activities that need to be completed at a certain time or after a certain amount of time has elapsed; Kvavilashvili \& Ellis, 1996). Barr (2011) also found no differences between individuals with stroke $(n=22)$ and controls $(n=22)$, or significantothers $(n=20)$ on total PRMQ score. However, the PRMQ has two subscales, one measuring PM and the other RM failures, and these were not reported separately. As PM and RM are considered to have different neural bases, a firm conclusion about self-reported PM impairment after stroke cannot be drawn from Barr's findings.

Man et al. (2015) assessed self-reported PM after stroke utilising the Brief Assessment of Prospective Memory (BAPM; Man, Fleming, Hohaus, \& Shum, 2011), a shortened version of the CAPM (Shum \& Fleming, 2014) which includes IADL and BADL items. Individuals with stroke $(n=75)$ reported significantly more PM failures compared to controls $(n=112)$, however this finding was dependent on age. Older stroke participants reported more BADL PM failures than the younger controls, and more frequent IADL PM 
failures than the young stroke, young control, and older control groups. No differences were found between individuals with stroke and their significant-others $(n=65)$ on total PM. However, when the subscales were assessed separately it was found that relatives reported significantly more PM failures than stroke participants on the IADL subscale, indicating PM impairment was only impacting instrumental and not basic routine activities. These findings may be due to the larger sample size, and hence greater power, or due the fact that the BAPM may measure different aspects of PM compared to the PRMQ used in previous studies.

As the previous literature examining self-reported PM after stroke is both limited and shows mixed findings dependent on the measure used and how the results were reported, it is important that further research examines PM after stroke. Furthermore, it is important that research go beyond self-reports of PM failure frequency, to also examine how important individuals think these are, especially since PM is linked with independence in ADL. By comparing self-reported PM measures with the reports of significant-others, we may gain further insight into how individuals with stroke view the frequency and importance of PM lapses after stroke.

\section{Aims and Hypotheses}

This study aimed to compare the self-reported PM of individuals with stroke to neurologically healthy controls, to determine if PM is impaired after stroke. Instead of using a single self-report questionnaire, both the PRMQ and BAPM were used to ensure that the previous findings were not questionnaire dependent. When using the PRMQ, separate RM and PM subscale scores were examined to avoid the confound of grouping the two scores together (as in Barr's study; 2011). It was hypothesised that there would be a significant difference between the stroke and control group on both the BAPM and PRMQ. The following research question was formulated: Does self-reported PM differ between 
individuals with stroke and healthy controls, and is this dependent on the specific measure used to assess self-reported PM? Additionally, the current study aimed to compare the selfreported PM of individuals with stroke to significant-others, to ascertain the level of selfawareness individuals with stroke have into their own PM function. Based on the previous results from Man, Yip, et al. (2015) it was also hypothesised that individuals with stroke would report less PM impairment than significant-others on Part A of the BAPM, particularly on the IADL subscale. No previous research has used Part B of the BAPM in the assessment of PM after stroke, therefore no hypothesis was formulated. Rather the following research question was posed: Do individuals with stroke report PM forgetting to be more problematic compared to healthy controls and their significant-others? Lastly, the study aimed to examine the relationships between the BAPM and PRMQ to see if they were highly associated with one another, providing evidence of concurrent validity. It was further hypothesised that strong correlations would exist between the two self-reported PM measures, particularly for the BAPM and PM subscale of the PRMQ.

\section{Method}

\section{Participants}

Twenty-eight individuals with stroke (17 females; 18 first-time stroke; aged 34-83), 25 significant-others (14 females), and 27 neurologically healthy controls ( 20 females; aged 44-79) took part in the current study, as part of a larger study looking at PM after stroke. Individuals with stroke were recruited through multiple health facilities and stroke organisations in South-East Queensland, Australia. All participants were required to be aged between 18 and 85 years, with a diagnosis of cerebrovascular accident within the last five years, and living in the community post-stroke for at least one month. Exclusion criteria included: stroke located in the cerebellum/brainstem; history of previous brain 
injury/neurological illness (other than stroke); diagnosed dementia/neurodegenerative illness; significant psychiatric disorder (e.g., schizophrenia and bipolar); insufficient communicative ability (i.e., severe aphasia); significant visual, hearing or hand function impairment that would impact on participation ability; or current alcohol/substance abuse.

Time post-stroke ranged from 2 to 53 months $(M=16.13, S D=13.82)$, while duration of living in the community post-stroke ranged from 1 to 52 months $(M=14.80, S D=12.93)$. Stroke site of lesion was left hemisphere for 11 cases, right hemisphere for 11, bilateral for 5, and 1 with unknown lateralisation. The type of stroke was documented as ischaemic in 19 cases, 7 were haemorrhagic, and 2 involved both ischaemic and haemorrhagic mechanisms. Disability was measured at recruitment using the Modified Rankin Scale (MRS; Rankin, 1957) with 8 individuals with no disability, 11 slight disability, 7 moderate disability, and 2 moderately severe disability. Overall participants reported high levels of daily functioning ( $M$ $=52.68, S D=14.37)$ on the Nottingham Extended Activities of Daily Living Scale (NEADL; Nouri \& Lincoln, 1987) completing most tasks independently or independently with difficulty.

Controls were recruited through Griffith University (subject-pool, Learning Space, and research volunteer email) and community groups. Inclusion criteria included: aged 18 to 85 years; and no history of neurological illness, brain injury, or stroke. Participants were excluded if they had a significant psychiatric illness, current alcohol/substance abuse, significant visual/hearing impairment, or insufficient communicative ability. Individuals with stroke and controls did not differ on any demographic variables (all $p<.05$, see Table 1 ) or level of depression, anxiety, or stress measured by the Depression, Anxiety, and Stress Scale - 21(DASS-21; Henry \& Crawford, 2005). 
Twenty-five significant-others (i.e., relative, spouse, friend, etc.) of various relationships (16 spouse/partners, 3 siblings, 3 friends, 2 children, and 1 other relative) were recruited alongside individuals with stroke. They had a mean age of 58.08 years $(S D=17.07)$ and mean education of 14.07 years $(S D=2.28)$. Significant-others had known the individuals with stroke between 1 to 65 years $(M=32.08, S D=20.04)$ and the majority were in contact with the individual with stroke everyday $(n=20)$ with others in contact 5 - 6 days per week $(n=1), 3$ - 4 days per week $(n=2), 1-2$ days per week $(n=1)$ or less than 1 day per week $(n$ $=1$ ). Ten significant-others were extremely confident, seven very confident, seven fairly confident, and one somewhat confident in rating the memory abilities of the individual with stroke. Frequency of contact was significantly positively correlated with rating confidence $(r$ $=.677, p<.001)$.

\section{Measures}

Participants completed two PM questionnaires. The PRMQ (Smith et al., 2000) is a 16-item self-reported measure examining both PM and RM failures in everyday life. Participants are required to rate how often they have memory failures for things that happened in the past and things that need to happen in the future. Responses range from never (1) to very often (5), with higher scores indicating more memory lapses. A total score is calculated for each subscale (retrospective and prospective) which range between 8 and 40 . A proxy-version is available, wherein a significant-other can rate the memory failures of another individual (Crawford, Henry, Ward, \& Blake, 2006). The PRMQ has previously been found to have good internal consistency when used on a non-clinical sample for the total, PM, and RM scores ( $\alpha=0.89,0.84,0.80$, respectively; Crawford, Smith, Maylor, Della Sala, \& Logie, 2003), and has previously been used with stroke populations (Kim et al., 2009). 
The BAPM (Man et al., 2011) is a self-reported questionnaire and consists of a Part A and B. Part A assesses the frequency of PM failure within the last month across 16 items. There are three BAPM scores, including the total score, and BADL and IADL subscale scores. Responses can range from never (1) to very often (daily; 5), with higher scores indicating more lapses in PM. Participants may also respond not applicable $(N / A)$ if they do not complete a certain task in their everyday lives. Part B uses the same items, however the question changes to how much of a problem would it be if you did forget to complete the task. Both total, and BADL and IADL subscale scores are calculated (mean score not including items answered $N / A$ ) with responses ranging from no problem at all (1) to a very serious problem (5). A proxy-version is available for significant-others. The BAPM has been used in stroke populations previously (Man et al., 2015) and has good reliability and validity when used with a traumatic brain injury (TBI) sample (Man et al., 2011). Across two healthy control samples ( $n=527$ and 95, respectively) reliability for the Part A BAPM total score ranged from 0.84 to 0.83 indicating acceptable internal consistency. In addition, the BADL (0.69 to 0.79$)$ and IADL ( 0.76 to 0.77$)$ subscale scores had good to acceptable internal consistency. In a sample of 45 patients with TBI, internal consistency ranged from strong to acceptable for the total $(\alpha=0.90)$, BADL $(\alpha=0.74)$, and IADL scores $(\alpha=0.76)$.

Additionally, support for the content and concurrent validity of the BAPM was found for the two healthy control groups with correlations between the BAPM and CAPM (Shum \& Fleming, 2014) all reaching 0.9 or above. Similar results were found for the TBI sample, where correlations were 0.89 . Test-retest reliability was tested using a subset of one of the healthy control samples $(n=26)$ and was found to have acceptable reliability for both the IADL $(\mathrm{ICC}=0.76)$ and BADL $(\mathrm{ICC}=0.66)$ subscales. 


\section{Procedure}

Ethical clearance was obtained from the relevant hospital and university ethics committees prior to study commencement. Written consent was obtained by all participants before starting the first session. The current study was conducted as part of a larger study; therefore, a neuropsychological battery was conducted over two sessions, each taking approximately 1.5 to 2 hrs to complete. The sessions were held in the participants' homes or in a quiet room at the university. After the completion of both sessions, participants were compensated $\$ 20$, or 2 credit points if recruited through the Griffith subject-pool. The two self-report questionnaires were completed in the first session. If a significant-other was able to attend the session, they were asked to complete the proxy-versions of the questionnaires at that point. If they were unable to attend, the questionnaires were posted with completion instructions, and a reply-paid envelope for its return.

\section{Statistical Analysis}

Data were analysed using IBM SPSS Statistics for Windows (Version 25; IBM Corporation, 2017). Data were screened for accuracy, missing values, outliers, and normality. The BAPM Part A BADL subscale was significantly positively skewed. Transformations were applied to address the skew however this did not change the significance of the results, therefore, the untransformed scores are reported. Comparisons of the BAPM scores (BADL, IADL, and total for Parts A and B) and PRMQ scores (PM and RM) were made between stroke and control groups, and between the stroke and their significant-others using independent samples $t$-tests. Effect sizes were determined using Cohen's $d$ and interpreted using the following criteria: small $=0.2$; medium $=0.5$; and large $=0.8(\mathrm{Hu}, 2010)$. Pearson's correlation coefficient was used to examine the relationships between scores on the two selfreport questionnaires for the groups separately. Effect sizes for the correlations were 
interpreted using the following criteria: small $=0.1$, medium $=0.3$, and large $=0.5$ (Cohen, 1998).

\section{Results}

\section{Comparison Between Stroke and Control Participants}

Independent sample $t$-tests were used to compare scores on the BAPM and PRMQ between individuals with stroke and controls (Table 2). On Part A of the BAPM, individuals with stroke reported significantly more failures in overall PM compared to controls (medium effect size). When broken into subscales, individuals with stroke reported significantly more PM failures than controls on BADL (medium effect size), but not the IADL subscale. The means for both groups fell between the never to rarely forgetting range for all subscales. On Part B of the BAPM, the results were similar for the two groups on the total score and the IADL subscale. However, individuals with stroke reported BADL PM failures as significantly less problematic or important than controls (medium effect size), with means for both groups falling between the slight to moderate problem range for all subscales.

Insert Table 2

When assessed using the PRMQ, individuals with stroke reported significantly more forgetting on both the RM and PM subscales compared to controls (medium effect sizes for both comparisons). The means fell between the rarely to sometimes forgetting range for both groups on the PM subscale, and the RM subscale for the individuals with stroke. For controls, the group mean for the RM subscale fell between the never to rarely forgetting range.

\section{Comparison Between Significant-other and Self-reports}

Independent sample $t$-tests were used to compare scores on the BAPM and PRMQ between individuals with stroke and their significant-others (Table 3). While the significant- 
others reported more frequent memory failures and importance of failures than individuals with stroke on the total and subscale scores of the BAPM Part A and B, these differences were not statistically significant. For individuals with stroke, both the total PM, IADL, and BADL subscales for Part A fell between the never to rarely forgetting range. While the significant-others means fell between the same ranges for the total and BADL subscale, their mean for the IADL subscale fell between the rarely to occasionally range. On Part B of the BAPM, both individuals with stroke and significant-others means on all three scales (total, IADL, and BADL) fell between the slight to moderate problem range. Individuals with stroke reported higher forgetting on both the PM and RM subscales of the PRMQ compared to their significant-others, however these differences were not statistically significant. Mean scores for both groups on each subscale fell between the rarely to sometimes forgetting range. -Insert Table 3

\section{Correlational Analyses}

Correlational analyses were conducted to examine the relationships between the BAPM and PRMQ (Table 4). For individuals with stroke, the PRMQ PM subscale was significantly correlated with all three subscales of the BAPM Part A. Similar results were found for the controls except for the BADL subscale. The RM subscale was significantly correlated with the BAPM Part A Total and IADL subscale, but not the BADL subscale for both individuals with stroke and controls. Additionally, the PRMQ PM subscale was significantly correlated with all three subscales of the BAPM Part B. However, no significant correlations were found for the RM subscale and Part B of the BAPM. No significant correlations were found for controls between the BAPM Part B and the PRMQ. 


\section{Discussion}

This study aimed to compare the self-reported PM of individuals with stroke to neurologically healthy controls and their significant-others to determine if PM is impaired after stroke. Analyses revealed that individuals with stroke reported significantly more total PM impairment compared to controls on Part A of the BAPM, supporting the hypothesis. When broken into subscales, they reported more PM failures compared to controls on both subscales, however statistical significance was only reached for the BADL subscale. On Part B of the BAPM, individuals with stroke reported memory failures as less problematic or important compared to controls on all three scores, however only the BADL subscale reached statistical significance. On the PRMQ, individuals with stroke reported significantly more forgetting than controls on both the PM and RM subscales, supporting the hypothesis. The research question that was formulated for this study asked: Does self-reported PM differ between individuals with stroke and healthy controls, and is this dependent on the specific measure used to assess self-reported PM? The current findings suggest that self-reported PM does differ between individuals with stroke and healthy controls, with some variation in performance on the measures. These findings further highlight that self-reported PM performance differs dependent on the measure used and the specific aspect of PM that the questionnaires are tapping into.

When using the BAPM Part A, the current study found individuals with stroke reported more PM impairments than controls overall, particularly for BADL. These findings are consistent with previous findings by Man et al. (2015), who found that individuals with stroke reported more PM failures on the BADL subscale than controls. However, this was only for their older participants, whereas the current finding is inclusive of the total stroke sample. The previous research also found differences between the groups on the IADL subscale which is not consistent with the current findings. These contrasting findings may be 
due to the larger sample size in the study by Man, Yip, et al. In contrast to previous research (Barr, 2011; Kim et al., 2009; Miller \& Radford, 2014), the current study found significant differences between the individuals with stroke and controls on the PM and RM subscales of the PRMQ. Individuals with stroke reported significantly more PM and RM failures compared to controls. The inconsistent finding may be due to the fact that the current research reported the subscales separately.

The current study is the only one to date to report the data from individuals with stroke on Part B of the BAPM, which measures self-perceived importance of PM failures. While individuals with stroke reported more PM failures compared to controls in Part A, when asked about how much of a problem this type of forgetting is in Part B, they reported that it was less of a problem or important, compared to controls. It could be argued that those with stroke seem to underestimate the importance of PM impairment. Possibly because some individuals with stroke have carers or significant-others to take care of these activities for them, therefore, relying on someone else to prompt them to complete the task. Particularly if individuals have a high level of disability and can no longer complete the tasks independently. Additionally, if individuals with stroke also have significant language, visual, or physical impairments (Gresham et al., 1995; Stroke Foundation, 2017), they may be more focused on these problems and how they impact their daily lives, rather than focusing on their memory as a key concern. The current sample did include individuals with stroke with mild to moderate language and physical impairments, and only those with significant impairments that would impact on testing performance were excluded from the study. Additionally, anecdotal evidence suggested that some individuals in the study were more concerned with other impairments impacting on their daily lives (i.e., communication, mobility), rather than their memory impairment. Therefore, their lack of motivation at improving their memory ability may have played an impact on their reporting of memory deficits. 
It could be possible that the individuals that rate their memory lapses as less important are applying a self-defence mechanism. Individuals with stroke reported more failures, but then reported that it does not really matter that they forget these tasks, possibly employing a self-defence mechanism to protect themselves. PM failures can cause great embarrassment or significant negative social consequences for individuals (Winograd, 1988). For example, if you asked an individual to name the former Prime Minister and they could not remember their name, no significant harm would be caused, and others would most likely credit the failure to the fact that their memory is slightly unreliable. However, if you asked that same person to post an important letter for you and they forgot (PM failure), you or other people may conclude that the person is unreliable, rather than attribute their behaviour to a lapse in memory. In terms of BADL, if someone forgets to shower themselves and is malodourous, others may conclude that the individual is unhygienic and may not want to be near them.

Given the findings of the current study, it is important that clinicians pay attention not only to individuals' physical and language deficits after stroke, but also assess whether impairments in PM are apparent. If PM impairment is present, it is important that individuals with stroke also understand the possible consequences of such an impairment. If individuals understand how significant PM is to their everyday life and in some circumstances their safety as well as the safety of others (e.g., forgetting to turn off an iron could cause a fire or forgetting to take medication could lead to another stroke), more focus could be placed on their rehabilitation which aims to improve PM ability. If it is assumed that the individual does not think something is a problem for them, then he or she would not spend much time or focus their attention/resources on trying to improve it.

The current study also aimed to ascertain a level of self-awareness individuals with stroke have into their own PM abilities by comparing self-reports to the reports of their significant-others. It was hypothesised that individuals with stroke would report less PM 
impairment when compared to their significant-others, particularly on the IADL subscale of the BAPM Part A. While there were slight differences in the reporting between the groups, no significant differences were found on any score of the BAPM or PRMQ, therefore, the hypothesis was not supported by the current findings. These results suggest accurate selfawareness of PM ability after stroke. This is in agreement with some of the previous studies (Barr, 2011) but not others (Man et al., 2015). It is also important to note that the current stroke sample, when tested using a psychometrically valid clinical measure (Cambridge Prospective Memory Test; CAMPROMPT; Wilson et al., 2005) as part of the larger study, performed significantly lower than controls, suggesting the presence of PM impairment (Hogan, Cornwell, Fleming, \& Shum, 2020). These findings are consistent with the selfreport of more PM failures when compared to controls in the current study.

It is important to note that self-awareness was not assessed separately, therefore we can only make assumptions on PM ability based on the comparison between individuals with stroke and their significant-others, which has limitations of its own as previously outlined. Individuals with acquired brain injury often show impaired ability to accurately perceive their own impairments as well as understand the impact that these deficits have on their everyday functioning (Caldwell et al., 2014). On the other hand, while impairments in self-awareness are often apparent after stroke (Hartman-Maeir, Soroker, Oman, \& Katz, 2003), not everyone will show impairments, as it is highly dependent on the location of stroke. Using a standardised and psychometrically valid and reliable measure of self-awareness (i.e., Awareness Questionnaire; Sherer, Bergloff, Boake, High Jr, \& Levin, 1998) would allow us to draw stronger conclusions on an individual's ability to report their own PM. Especially since self-report methods can be subject to rater bias, wherein the individual reports higher or lower levels of ability in order to make themselves appear in a negative or positive way (Hogan et al., 2016; Kliegel \& Jager, 2006; Meeks, Hicks, \& Marsh, 2007). 
Lastly, the current study aimed to examine the relationships between the BAPM and PRMQ. Significant correlations were found between the PM subscale of the PRMQ and total BAPM score for individuals with stroke and controls, supporting the hypothesis. Both subscale scores (BADL and IADL) and the total score of the BAPM (Parts A and B) significantly correlated with the PRMQ PM subscale for the individuals with stroke, and the total and IADL subscale for the controls. Some subscales of the BAPM Part A also correlated with the RM subscale of the PRMQ. However, higher correlations were found for the prospective component over the retrospective score. These findings support the concurrent validity of the BAPM in assessing self-reported PM ability after stroke. Possibly, PM failures for more routine BADL are not reliant on the same mechanisms as PM for more complex IADL and RM. These could possibly be due to lapses in attention rather than memory failures, however more research is needed to confirm this suggestion.

Overall, the current study was limited by the small sample size and the heterogeneity of the sample (i.e., time since stroke onset, severity and location of stroke). It could be argued that significant-others completing proxy-versions may not be fully aware of their relatives' daily memory function. The only way for significant-others to be aware of every PM failure, is if the individual with stroke shares every prospective intention with them, which is highly unlikely, particularly for those who do not live with their significant-other. As self-reported questionnaires do not observably measure PM performance (Uttl \& Kibreab, 2011), it is important that research examining PM performance using self-reported methods be interpreted with caution and limitations taken into consideration (Barr, 2011; Brooks et al., 2004; Kim et al., 2009; Man et al., 2015) and it is recommended that self-report measures be utilised in conjunction with standardised objective measures of PM.

Despite the limitations, the current research has provided a clearer picture of selfreported PM after stroke by using more than one questionnaire and reporting the subscales 
separately. By using multiple PM questionnaires that tap into different aspects of PM and reporting the subscales separately, the current study has helped to clarify and strengthen the findings regarding self-reported PM after stroke. Self-reported PM measures are quick and easy to administer, providing clinicians/researchers with a brief screening tool to see if further PM assessment is required. We can also gain valuable insight into how clinical populations both see their own PM ability and how problematic they think the memory failures are. The information garnered from these questionnaires could help to shape future PM rehabilitation, not only to help improve PM performance but also to teach individuals the importance of PM ability in daily life and the possible consequences of PM lapses. Therefore, future research should be conducted to examine the PM abilities of individuals with stroke by utilising both self-reported questionnaires as well as neuropsychological measures of PM to gain a greater understanding of how PM changes after stroke and its impact on daily living.

Funding details: This work was supported by a Griffith University Post-graduate Scholarship

Disclosure statement: The authors report no conflict of interest. 


\section{References}

Andrews, G., Halford, G. S., Shum, D. H., Maujean, A., Chappell, M., \& Birney, D. P. (2014). Verbal learning and memory following stroke. Brain Injury, 28(4), 442-447. doi:10.3109/02699052.2014.888758

Barr, A. C. (2011). Prospective memory functioning after stroke: A research portfolio. (Doctoral thesis, University of Edinburgh, Scotland). Retrieved from https://www.era.lib.ed.ac.uk/handle/1842/6049.

Brooks, B. M., Rose, F. D., Potter, J., Jayawardena, S., \& Morling, A. (2004). Assessing stroke patients' prospective memory using virtual reality. Brain Injury, 18(4), 391340. doi:10.1080/02699050310001619855

Caldwell, S. B., Wilson, F. C., McBrinn, J., Carton, S., Delargy, M., McCann, J. P., ... McGuire, B. E. (2014). Self-awareness following acquired brain injury: measurement and relationship to executive functioning. The Irish Journal of Psychology, 35(2), 5368. doi:10.1080/03033910.2014.921228

Cohen, J. (1998). Statistical power analysis for the behavioural sciences ( $2^{\text {nd }}$ ed.). New Jersey: Lawrence Erlbaum.

Crawford, J., Smith, G., Maylor, E., Della Sala, S., \& Logie, R. (2003). The prospective and retrospective memory questionnaire (PRMQ): Normative data and latent structure in a large non-clinical sample. Memory, 11(3), 261-275. doi:10.1080/09658210244000027

Crawford, J., Henry, J. D., Ward, A. L., \& Blake, J. (2006). The prospective and retrospective memory questionnaire (PRMQ): Latent structure, normative data and discrepancy analysis for proxy-ratings. British Journal of Clinical Psychology, 45(1), 83-104. doi:10.1348/014466505X28748 
de Haan, E. H., Nys, G. M., \& Van Zandvoort, M. J. (2006). Cognitive function following stroke and vascular cognitive impairment. Current Opinion in Neurology, 19(6), 559564. doi:10.1097/01.wco.0000247612.21235.d9

Ekstam, L., Uppgard, B., Kottorp, A., \& Tham, K. (2007). Relationship between awareness of disability and occupational performance during the first year after a stroke. American Journal of Occupational Therapy, 61(5), 503-511. doi:10.5014/ajot.61.5.503

Fish, J., Wilson, B. A., \& Manly, T. (2010). The assessment and rehabilitation of prospective memory problems in people with neurological disorders: A review. Neuropsychological Rehabilitation, 20(2), 161-179. doi:10.1080/09602010903126029

Gresham, G. E., Duncan, P. W., Stason, W. ., Adams, H. P., Adelman, A. M., Alexander, D. N., ... Trombly, C. A. (1995). Post-stroke rehabilitation. Clinical Practice Guideline, No. 16 U.S. Department of Health and Human Services. Public Health Service, Agency for Health Care Policy and Research. AHCPR Publication No. 95-0662.

Groot, Y. C. T., Wilson, B. A., Evans, J., \& Watson, P. (2002). Prospective memory functioning in people with and without brain injury. Journal of the International Neuropsychological Society, 8(2), 645-654. doi:10.1017/S1355617702801321

Hartman-Maeir, A., Soroker, N., Ring, H., \& Katz, N. (2002). Awareness of deficits in stroke rehabilitation. Journal of Rehabilitation Medicine, 34(4), 158-164. doi:10.1080/16501970213236

Hartman-Maeir, A., Soroker, N., Oman, S. D., \& Katz, N. (2003). Awareness of disabilities in stroke rehabilitation - A clinical trial. Disability and Rehabilitation, 25(1), 35-44. doi:10.1080/0963828021000007897 
Henry, J. D., \& Crawford, J. R. (2005). The short-form version of the depression anxiety stress scales (DASS-21): Construct validity and normative data in a large non-clinical sample. British Journal of Clinical Psychology, 44(2), 227-239. doi:10.1348/014466505X29657

Hogan, C., Fleming, J., Cornwell, P., \& Shum, D. (2016). Prospective memory after stroke: A scoping review. Brain Impairment, 17(2), 123-142. doi:10.1017/BrImp.2016.12

Hogan, C., Cornwell, P., Fleming, J., \& Shum, D. H. K. (2020). The assessment prediction of prospective memory after stroke. Journal of the International Neuropsychological Society. 1-10. doi: 10.1017/S1355617720000405

Hu, Q. (2010). Effect size, measures of. In N. J. Salkind (Ed.), Encyclopedia of research design (pp. 408-412). Thousand Oaks, CA: SAGE Publications, Inc.

IBM Corporation. (2017). IBM SPSS Statistics (Version 25) [Computer Software]. New York: IBM Corporation.

Kalashnikova, L., Zueva, Y. V., Pugacheva, O., \& Korsakova, N. (2005). Cognitive impairments in cerebellar infarcts. Neuroscience and Behavioral Physiology, 35(8), 773-779. doi:10.1007/s11055-005-0123-0

Kim, H. J., Craik, F., Luo, L., \& Ween, J. E. (2009). Impairments in prospective and retrospective memory following stroke. Neurocase, 15(2), 145-156. doi:10.1080/13554790802709039

Kliegel, M., \& Jager, T. (2006). Can the prospective and retrospective memory questionnaire (PRMQ) predict actual prospective memory performance? Current Psychology: A Journal for Diverse Perspectives on Diverse Psychological Issues, 25(3), 182-191. doi:10.1007/s12144-006-1002-8 
Kliegel, M., McDaniel, M. A., \& Einstein, G. O. (Eds.). (2008). Prospective memory: Cognitive, neuroscience, developmental, and applied perspectives. New York: Lawrence Erlbaum Associates, Inc.

Kvavilashvili, L., \& Ellis, J. (1996). Varieties of intention: Some distinctions and classifications. In M. Brandimonte, G. O. Einstein, \& M. A. McDaniel (Eds.), Prospective memory: Theory and applications (pp. 23-51). New Jersey: Lawrence Erlbaum Associates, Inc.

Leung, D. P. K., \& Liu, K. P. Y. (2011). Review of self-awareness and its clinical application in stroke rehabilitation. International Journal of Rehabilitation Research, 34(3), 187195. doi:10.1097/MRR.0b013e3283487f31

Man, D. W. K., Fleming, J., Hohaus, L., \& Shum, D. (2011). Development of the brief assessment of prospective memory (BAPM) for use with traumatic brain injury populations. Neuropsychological Rehabilitation, 21(6), 884-898. doi:10.1080/09602011.2011.627270.

Man, D. W. K., Yip, C., Lee, G., Fleming, J., \& Shum, D. (2015). Self-report prospective memory problems in people with stroke. Brain Injury, 29(3), 329-335. doi:10.3109/02699052.2014.974672.

Meeks, J. T., Hicks, J. L., \& Marsh, R. L. (2007). Metacognitive awareness of event-based prospective memory. Consciousness and Cognition, 16(4), 997-1004. doi:10.1016/j.concog.2006.09.005

Miller, L. A., \& Radford, K. (2014). Testing the effectiveness of group-based memory rehabilitation in chronic stroke patients. Neuropsychological Rehabilitation, 24(5), 721-737. doi:10.1080/09602011.2014.894479

Nouri, F. M., \& Lincoln, N. B. (1987). An extended activities of daily living scale for stroke patients. Clinical Rehabilitation, 1(4), 301-305. doi:10.1177/026921558700100409 
Ownsworth, T., \& Clare, L. (2006). The association between awareness deficits and rehabilitation outcome following acquired brain injury. Clinical Psychology Review, 26(6), 783-795. doi:10.1016/j.cpr.2006.05.003

Radford, K. A., Lah, S., Say, M. J., \& Miller, L. A. (2011). Validation of a new measure of prospective memory: The royal prince alfred prospective memory test. The Clinical Neuropsychologist, 25(1), 127-140. doi:10.1080/13854046.2010.529463

Rankin, J. (1957). Cerebral vascular accidents in patients over the age of 60: II. prognosis. Scottish Medical Journal, 2(5), 200-215. doi:10.1177/003693305700200504

Rendell, P. G., \& Henry, J. D. (2009). A review of virtual week for prospective memory assessment: Clinical implications. Brain Impairment, 10(1), 14-22. doi:10.1375/brim.10.1.14

Shere, M., Bergloff, P., Boake, C., High Jr, W., \& Levin, E. (1998). The awareness questioannire: Factor structure and internal consistency. Brain Injury, 12(1). 63-68. doi:10.1080/026990598122863

Shum, D., Fleming, J., \& Neulinger, K. (2002). Prospective memory and traumatic brain injury: A review. Brain Impairment, 3(1), 1-16. doi:10.1375/brim.3.1.1

Shum, D., Levin, H., \& Chan, R. C. K. (2011). Prospective memory in patients with closed head injury: A review. Neuropsychologia, 49(8), 2156-2165. doi:10.1016/j.neuropsychologia.2011.02.006

Shum, D., \& Fleming, J. (2014). Comprehensive assessment of prospective memory [Clinical assessment and user manual]. Gold Coast: Griffith Health Institute

Smith, G., Del Sala, S., Logie, R. H., \& Maylor, E. A. (2000). Prospective and retrospective memory in normal ageing and dementia: A questionnaire study. Memory, 8(5), 311321. doi:10.1080/09658210050117735 
Stroke Foundation. (2017). Clinical guidelines for stroke management 2017. Stroke Foundation.

Uttl, B., \& Kibreab, M. (2011). Self-report measures of prospective memory are reliable but not valid. Canadian Journal of Experimental Psychology/Revue Canadienne De Psychologie Experimentale, 65(1), 57-68. doi:10.1037/a0022843

Wilson, B., Shiel, A., Foley, J., Emslie, H., Groot, Y., Hawkins, K., \& Watson, P. (2005). Cambridge test of prospective memory (CAMPROMPT). San Antonio: Pearson Assessment.

Winograd, E. (1988). Some observations on prospective remembering. In M. M. Gruneberg, P. E. Morris, \& R. N. Sykes (Eds.), Practical aspects of memory: Current research and issues (Vol. 1, pp. 348-353). Chichester, England: John Wiley.

Woods, S. P., Weinborn, M., Velnoweth, A., Rooney, A., \& Bucks, R. S. (2012). Memory for intentions is uniquely associated with instrumental activities of daily living in healthy older adults. Journal of the International Neuropsychological Society, 18(1), 134-138. doi:10.1017/S1355617711001263 
Table 1

Demographic Information for Individuals with Stroke $(n=28)$ and Healthy Controls $(n=27)$

\begin{tabular}{|c|c|c|c|c|c|}
\hline \multirow[b]{3}{*}{ Variable } & Individuals with & & \multirow[b]{3}{*}{$t$} & \multirow[b]{3}{*}{$d f$} & \multirow[b]{3}{*}{$p$} \\
\hline & stroke & Controls & & & \\
\hline & $M(S D)$ & $M(S D)$ & & & \\
\hline Age (years) & $62.86(13.05)$ & $56.41(10.69)$ & 2.00 & 53 & .051 \\
\hline Education (years) & $14.09(4.77)$ & $14.26(4.77)$ & -0.15 & 48.28 & .878 \\
\hline Pre-morbid IQ & $103.75(12.94)$ & $105.37(11.34)$ & -0.49 & 53 & .624 \\
\hline Line Bisection & $15.64(2.25)$ & $16.44(0.93)$ & -1.72 & 53 & .092 \\
\hline \multicolumn{6}{|l|}{ DASS-21 } \\
\hline Depression & $4.00(4.07)$ & $2.89(3.70)$ & 1.06 & 53 & .295 \\
\hline Anxiety & $2.86(2.97)$ & $2.22(2.52)$ & 0.86 & 53 & .397 \\
\hline Stress & $5.57(4.75)$ & $4.67(3.39)$ & 0.81 & 53 & .421 \\
\hline
\end{tabular}

Note. DASS-21 = Depression, Anxiety, and Stress Scale -21. 
Table 2

Descriptive and Inferential Statistics for Self-reported Data in Individuals with Stroke $(n=$ 28) and Neurologically Healthy Controls $(n=27)$

\begin{tabular}{|c|c|c|c|c|c|c|}
\hline & Individuals & Controls & & & & \\
\hline & $M(S D)$ & $M(S D)$ & $t$ & $d f$ & $p$ & $d$ \\
\hline \multicolumn{7}{|c|}{ BAPM Part A } \\
\hline Total & $1.60(0.46)$ & $1.37(0.32)$ & 2.08 & 48.26 & $.04 *$ & 0.58 \\
\hline IADL & $1.81(0.57)$ & $1.59(0.45)$ & 1.58 & 53 & .12 & 0.43 \\
\hline BADL & $1.42(0.50)$ & $1.17(0.27)$ & 2.34 & 41.91 & $.02 *$ & 0.62 \\
\hline
\end{tabular}

BAPM Part B

$\begin{array}{lllllll}\text { Total } & 2.25(0.71) & 2.57(0.82) & -1.52 & 53 & .13 & -0.42 \\ \text { IADL } & 2.26(0.77) & 2.37(0.76) & -0.55 & 53 & .58 & -0.14 \\ \text { BADL } & 2.25(0.76) & 2.74(0.98) & -2.06 & 53 & .04 * & -0.56\end{array}$

PRMQ

$\begin{array}{lllllll}\text { Prospective } & 21.32(6.92) & 18.15(4.50) & 2.02 & 46.55 & .049 * & 0.54\end{array}$

$\begin{array}{lllllll}\text { Retrospective } & 18.36(5.40) & 15.59(4.47) & 2.06 & 53 & .04 * & 0.56\end{array}$

Note. ${ }^{*} p<.05 . \mathrm{BAPM}=$ Brief Assessment of Prospective Memory; IADL $=$ instrumental activities of daily living; $\mathrm{BADL}=$ basic activities of daily living; $\mathrm{PRMQ}=$ Prospective and Retrospective Memory Questionnaire. 
Table 3

Descriptive and Inferential Statistics for Self-reported Data in Individuals with Stroke $(n=$ 28) and Significant-others $(n=25)$

\begin{tabular}{|c|c|c|c|c|c|c|}
\hline & Individuals with & Significant-others & & & & \\
\hline & $M(S D)$ & $M(S D)$ & $t$ & $d f$ & $p$ & $d$ \\
\hline \multicolumn{7}{|l|}{ BAPM Part A } \\
\hline Total & $1.60(0.46)$ & $1.66(0.76)$ & -0.38 & 51 & .71 & -0.10 \\
\hline IADL & $1.81(0.57)$ & $2.15(1.22)$ & -1.28 & 51 & .19 & -0.36 \\
\hline BADL & $1.42(0.50)$ & $1.47(0.88)$ & -0.22 & 51 & .83 & -0.07 \\
\hline \multicolumn{7}{|l|}{ BAPM Part B } \\
\hline Total & $2.25(0.71)$ & $2.31(0.66)$ & -0.27 & 51 & .79 & -0.09 \\
\hline IADL & $2.26(0.77)$ & $2.46(0.86)$ & -0.90 & 51 & .37 & -0.25 \\
\hline BADL & $2.25(0.76)$ & $2.25(0.74)$ & -0.02 & 51 & .98 & 0.00 \\
\hline \multicolumn{7}{|l|}{ PRMQ } \\
\hline Prospective & $21.32(6.92)$ & $18.44(5.83)$ & 1.63 & 51 & .11 & 0.45 \\
\hline Retrospective & $18.36(5.40)$ & $17.60(6.08)$ & 0.48 & 51 & .63 & 0.13 \\
\hline
\end{tabular}

Note. BAPM = Brief Assessment of Prospective Memory; IADL = instrumental activities of daily living; $\mathrm{BADL}=$ basic activities of daily living; $\mathrm{PRMQ}=$ Prospective and Retrospective Memory Questionnaire. 
Table 4

Pearson's r Correlations Between BAPM and PRMQ

\begin{tabular}{|c|c|c|c|c|c|c|c|c|c|}
\hline & & \multicolumn{3}{|c|}{ BAPM A } & \multicolumn{3}{|c|}{ BAPM B } & \multicolumn{2}{|c|}{ PRMQ } \\
\hline & & Total & IADL & BADL & Total & IADL & BADL & $\mathrm{RM}$ & PM \\
\hline \multirow[t]{3}{*}{ BAPM A } & Total & - & $.87 *$ & $.86^{*}$ & $.64^{*}$ & $.63^{*}$ & $.58^{*}$ & $.59^{*}$ & $.75^{*}$ \\
\hline & IADL & $.94 *$ & - & $.49 *$ & $.66^{*}$ & $.59 *$ & $.64^{*}$ & $.57^{*}$ & $.71 *$ \\
\hline & BADL & $.84^{*}$ & $.60 *$ & - & .44 & $.49 *$ & .35 & .43 & $.57 *$ \\
\hline \multirow[t]{3}{*}{ BAPM B } & Total & .25 & .24 & .20 & - & $.93^{*}$ & $.94^{*}$ & .45 & $.73 *$ \\
\hline & IADL & .42 & .41 & .34 & $.93^{*}$ & - & $.76^{*}$ & .45 & $.75^{*}$ \\
\hline & BADL & .11 & .10 & .10 & $.96^{*}$ & $.81^{*}$ & - & .40 & $.65^{*}$ \\
\hline \multirow[t]{2}{*}{ PRMQ } & $\mathrm{RM}$ & $.54 *$ & $.57 *$ & .34 & .36 & .46 & .27 & - & $.74 *$ \\
\hline & PM & $.57 *$ & $.59^{*}$ & .37 & .32 & .41 & .25 & $.76^{*}$ & - \\
\hline
\end{tabular}

Note. ${ }^{*} p<.01$. Stroke group results above central line, controls below. BAPM $=$ Brief Assessment of Prospective Memory; IADL = instrumental activities of daily living; BADL = basic activities of daily living; PRMQ = Prospective and Retrospective Memory Questionnaire; $\mathrm{RM}=$ retrospective memory; $\mathrm{PM}=$ prospective memory. 